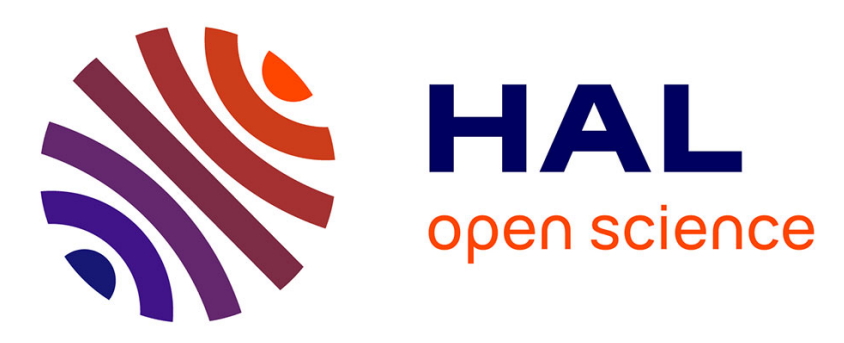

\title{
Multi-modal framework for subject-specific finite element model generation aimed at pressure ulcer prevention.
}

Marek Bucki, Yohan Payan, Francis Cannard, Bruno Diot, Nicolas Vuillerme

\section{To cite this version:}

Marek Bucki, Yohan Payan, Francis Cannard, Bruno Diot, Nicolas Vuillerme. Multi-modal framework for subject-specific finite element model generation aimed at pressure ulcer prevention.. Computer Methods in Biomechanics and Biomedical Engineering, 2013, 16 Suppl 1, pp.147-148. 10.1080/10255842.2013.815861 . hal-00862687

\section{HAL Id: hal-00862687 https://hal.science/hal-00862687}

Submitted on 18 Sep 2013

HAL is a multi-disciplinary open access archive for the deposit and dissemination of scientific research documents, whether they are published or not. The documents may come from teaching and research institutions in France or abroad, or from public or private research centers.
L'archive ouverte pluridisciplinaire HAL, est destinée au dépôt et à la diffusion de documents scientifiques de niveau recherche, publiés ou non, émanant des établissements d'enseignement et de recherche français ou étrangers, des laboratoires publics ou privés. 


\title{
Multi-Modal Framework for Subject-Specific Finite Element Model Generation aimed at Pressure Ulcer Prevention
}

\author{
Bucki M. ${ }^{1}$, Payan Y. ${ }^{2}$, Cannard F. ${ }^{1}$, Diot B. ${ }^{3}$, Vuillerme N. ${ }^{4,5}$ \\ ${ }^{1}$ TexiSense, Montceau-les-Mines, France \\ ${ }^{2}$ TIMC-IMAG Lab., UMR CNRS 5525, La Tronche, France \\ ${ }^{3}$ IDS, Montceau-les-Mines, France \\ ${ }^{4}$ Univ. Grenoble-Alpes, FRE 3405 AGIM Lab., CNRS-UJF-UPMF-EPHE, La Tronche, France \\ ${ }^{5}$ Institut Universitaire de France, Paris, France
}

Keywords: biomechanical modeling, finite element method, pressure ulcer prevention, mesh registration

\section{Introduction}

Pressure ulcers represent one of the most common, disruptive and disabling life threatening conditions affecting persons with spinal cord injury (SCI) and more largely wheelchair users. Whereas it can take only a few minutes for a pressure ulcer to develop, complete wound healing may require a months' hospital stay, involving difficult and expensive medical or surgical treatments. Currently available techniques and protocols designed to prevent pressure ulcer formation are mainly based on the improvement of the skin/support interface and on a postural and behavioral education. These techniques, however, seem to lack efficiency as the prevalence and incidence of pressure ulcers still remain very high. Development and validation of efficient solutions to prevent pressure ulcers is thus still strongly needed. Until recently, it was thought that deep tissue ulcers stemmed from internal overpressures yet recent results suggest that it is not the internal pressures, but the strains that best reflect the level of tissue damage [Loerakker et al.]. Internal strains can be estimated from the values of external interface pressures by resorting to biomechanical modeling in a Finite Element (FE) modeling framework. However, to properly estimate the levels of compression within the subject's soft tissues, the model must accurately replicate the considered morphology. This article describes a fast, automatic and robust technique for the generation of patient-specific models to be used within a personalized pressure ulcer prevention strategy. The technique resorts to complimentary modalities to gain insight at each patient's morphology while maintaining an acceptable benefits/risks (or costs) ratio.

\section{Methods}

A. Mesh warping: Mesh-Match-and-Repair To produce high quality personalized FE models, it is necessary to resort to medical imaging and acquire the most relevant possible description of the modeled morphology. Yet building a FE model from a medical data set can be a challenging and time consuming task. To overcome the commonly encountered problems - such as partial organ imaging, presence of noise or poorly reconstructed surfaces - the Mesh-Match-and-Repair (MMRep) "mesh warping" approach [Bucki et al.] has been chosen for its versatility. In this framework, a generic or "atlas" model representing a typical organ is first assembled. Then, for each patient the atlas model is warped, or registered, so that its shape accurately represents the target morphology. The atlas FE model was assembled using the Zygote (Zygote.com) data base comprising: pelvis (iliac bones and sacrum), femurs in seated position, skin surface and inner fat/muscle interface. MMRep is a four steps process. First, the patient data is registered onto the atlas. In this case, the patient's skin surface is fitted onto the atlas model's skin. Second, the resulting deformation is inverted in order to operate from atlas to patient frame. Thirdly, the deformation is applied to the atlas FE nodes (elements connectivity remains unchanged), bone model and fat/muscle interface. As the nonlinear deformation is computed based solely on the patient skin surface, the position of the inner structures is only a reasonable approximation of the actual patient's morphology.

\section{B. Multi-modal morphology acquisition}

An economically acceptable and practical image acquisition workflow must be designed in order to make personalized biomechanical modeling available for the largest number of wheelchair users. We propose to use two different modalities.

1. Kinect (Microsoft). This 3D surface scanner makes it possible to acquire within minutes the skin surface of the subject's buttocks. It is low-cost, easy to operate and does not present any risk for the subject. The "Reconstructme" software is used to convert the scanned outer shape of the buttocks into 3D triangular surface meshes (Reconstructme.net).

2. EOS bi-plane X-Ray imaging. This novel modality performs a full body scan with a radiation dose between 2.9 to 9.2 lower than a traditional XRay image. The output is 1:1 scale sagittal and frontal images for a $1.72 \mathrm{~m}$ high subject both with a 
pixel size of $0.18 \times 0.18 \mathrm{~mm}$. A very good contrast between soft and hard tissues permits to reconstruct in $3 \mathrm{D}$ the shape of the pelvic bones (iliac and sacrum).

\section{Model personalization}

For each subject, the set of medical images and surfaces is aligned with the atlas model using a set of 6 anatomical landmarks: left-anterior, leftposterior, right-anterior, right-posterior ischial spines, along with left and right trochanters. For the atlas, the landmarks are defined at atlas model assembly stage once and for all, and for each patient the landmarks are manually localized in each modality:

1. Kinect. Prior to surface scanning, the spines and trochanters are manually palpated and marked on the patient's skin using small plastic markers. Once the $3 \mathrm{D}$ surface reconstructed from the scan, the clearly visible markers are 'mouse-clicked'.

2. EOS. The same markers are used. The position of each marker in the EOS referential is found by identifying both sagittal and frontal projections of the considered marker.

First, a rigid registration computed on the set of 6 landmarks brings all patient data into the atlas reference frame. Then, a linear fit between patient and atlas landmarks compensates for most of the scale difference. Finally, the patient specific FE model is obtained by applying the MMRep nonlinear deformation.

\section{Results and Discussion}

A. Atlas model

The atlas FE mesh was produced using a hexahedral dominant meshing technique and comprises 14,868 elements. The pelvis and femurs are considered as fixed rigid bodies. The mechanical behavior of the fat and muscle tissues is modeled as an elastic Ogden material with parameters taken from [Oomens et al.], i.e. fat: $\mu=0.01 \mathrm{MPa}, \alpha=5$; and muscle: $\mu=0.003 \mathrm{MPa}, \alpha=30$.

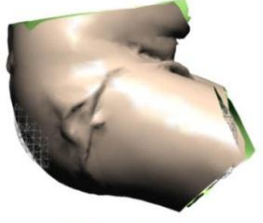

(a) Kinect output

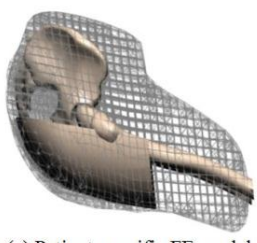

(c) Patient-specific FE model

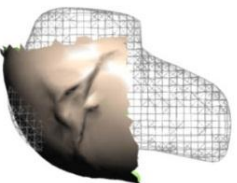

(b) Clipped skin surface and atlas model

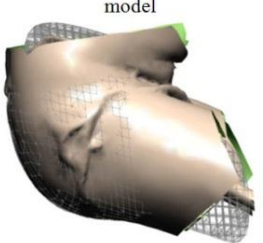

(d) Patient skin overlaid on FE model
Figure 1: FE model generation (sagittal view)

\section{B. FE model generation}

The accuracy of the Kinect device was initially assessed on spheres of known radius $(20 \mathrm{~cm})$. A mean error of 1.44 and a max error of $4.16 \mathrm{~mm}$ were obtained. The shape of the buttocks in 3 young healthy subjects was acquired after the 6 anatomical markers have been placed on the skin. The Kinect scan and surface reconstruction took approximately 2 minutes. Then, an EOS scanner was used to reconstruct the shape of the pelvis in each personalized FE model. In all three cases, a subjectspecific biomechanical model could be produced within 20 minutes (Fig. 1). Skin representation mean error was less than $1 \mathrm{~mm}$.

\section{Conclusions}

A framework for multi-modal generation of patient specific biomechanical models of the buttocks has been presented. This framework addresses the need for fast, automatic and robust model personalization in the context of pressure ulcer prevention for the wheelchair-ridden persons. It is also accurate as the representation error measured on the skin is less than $1 \mathrm{~mm}$ and the pelvis is modeled within its segmentation accuracy. The framework furthermore takes into account restrictions on the availability of medical images in situations where the benefits of the ulcer prevention strategy are deemed insufficient in regard of the imaging costs or incurred radiations. Indeed, a low-cost and radiation-free scenario based on a publicly available device (Microsoft Kinect) is proposed although resorting to it results in approximations and loss of accuracy. Finally, although the presented results are very promising, we must acknowledge that the relatively small sample size could constitute a limitation to the generalization of the current findings. Along these lines, the individual biomechanical modeling technique presented here will undergo validation on a large number of cases as part of an epidemiologic study carried out on 90 wheelchair users followed throughout 2013.

\section{Acknowledgments}

The authors wish to thank the ANR TecSan IDS project and CAMI Labex for their support.

\section{References}

S. Loerakker, E. Manders, G.J. Strijkers, K. Nicolay, F.P.T. Baaijens, D.L. Bader, C.W.J. Oomens, "The effects of deformation, ischaemia and reperfusion on the development of muscle damage during prolonged loading”, J. Appl. Phys., 111(4), pp. 1168-1177, 2011.

M. Bucki, C. Lobos, Y. Payan, “A Fast and Robust Patient Specific Finite Element Mesh Registration Technique: Application to 60 Clinical Cases", Med Image Anal, vol. 14, pp. 303-317, 2010.

C.W.J. Oomens, E.M.H. Bosboom, O.F.J.T. Bressers, C.V.C. Bouten, D.L. Bader, "Can loaded interface characteristics influence strain distribution in muscle adjacent to bony prominences", Comp. Meth. Biomech. Biomed. Eng. 6(3), pp. 171-180, 2003. 